\title{
The optimization of the tracking mechanism used for a group of PV panels
}

\author{
C. Alexandru, C. Pozna \\ Product Design and Robotics Department \\ University "Transilvania" of Braşov \\ 29 Bd. Eroilor, 500036 Braşov (Romania) \\ Phone/Fax:+40268472496, e-mail: calex@unitbv.ro, cp@unitbv.ro
}

\begin{abstract}
The paper presents researches on increasing the energetic \& economic efficiency of the photovoltaic conversion by designing and optimizing a dual-axis tracking mechanism that simultaneously changes the position of the panels using two motor sources; the driving source for the daily motion is a rotary motor, while the seasonal motion is performed with a linear actuator. The main task in optimizing the tracking mechanism is to maximize the energetic gain by increasing the solar input and minimizing the energy consumption for tracking. The design strategy aims to identify the optimum angular field for the daily motion, as well as the optimum actuating time in the step-by-step motion. The study is made by developing the virtual prototype of the tracking mechanism, in the mechatronic concept.
\end{abstract}

\section{Key words}

PV panels group, tracking mechanism, control system, virtual prototype, optimization.

\section{Problem Statement}

The paper is approaching a theme that belongs to a very important field: renewable sources for energy production - increasing the efficiency of the photovoltaic (PV) conversion. The researches in this field represent a priority at global level because provides viable alternatives to a series of major problems that humanity is facing: the limited and pollutant character of the fossil fuels, global warming or the greenhouse effect. The PV systems can deliver energy on large-scale to a competitive price is the conclusion of the European Commission for Energy, in the report "A Vision for Photovoltaic Technology for 2030 and Beyond" (2004). In this frame, the realization of the PV arrays (system of PV panels that function as a single electricity-producing unit) appeared as a necessity for the development of large systems for producing electric energy.

The energetic efficiency of the PV arrays depends on the degree of use of the solar radiation, which can be maximized by use of mechanical systems for the orientation of the panels in accordance with the paths of the Sun. Basically, the tracking systems are mechanical systems with $\mathrm{M}=1$ or $\mathrm{M}=2$ degrees of mobility (corresponding with the number of revolute axes), driven by rotary motors or linear actuators, which are controlled in order to ensure the optimal positioning of the panel relatively to the Sun position on the sky dome, on the entire period of the day (the diurnal motion, East - West), and also depending on the season (the seasonal/elevation motion). From energetic point of view, the photovoltaic array with tracking is efficient if the following condition is achieved: $\varepsilon=\left(E_{T}-E_{F}\right)-E_{C}>0$, where $E_{T}$ is the electric energy produced by the $\mathrm{PV}$ array with tracking, $E_{F}$ - the energy produced by the same array without tracking (fixed), and $\mathrm{E}_{\mathrm{C}}$ - the energy consumption for orienting the panels. In the current conditions, the maximization of the efficiency parameter $\varepsilon$ through the optimal design of the tracking system became an important challenge in the modern research and technology.

In practice, there are two solutions for developing the tracked arrays, depending on the existence (or not) of a common frame: (a) PV platforms, where the panels are mounted on a common frame (panels with same sustaining structure), orientation being realized simultaneously by the orientation of the entire platform; (b) array with individual panels, where the panels are separately mounted on individual sustaining structures. In the second case, the orientation can be realized in two ways: (b.1) independent orientation for each panel of the array (module with own orientation system - self motor source); (b.2) simultaneous orientation of all modules of the group, or tracking for groups of panels, by using single driving source (for single-axis systems) or two driving sources (for dual-axis systems), which transmits the motion to the all modules.

The simultaneous orientation (b.2), even is more complex by constructive aspects, ensures a greater energetic efficiency because of the minimization of the consumers in the array. This solution is also very useful from the economic efficiency point of view and this because the 
most expensive component of a PV system with tracking is the controlled motor source. At the same time, the reliability and the durability of the PV system is increasing as consequence of the minimization of the motor sources. The simultaneous orientation, with the predicted advantages and the characteristic problems, opens a research area insufficiently explored since now, fact sustained by the literature and practical developments in the field that refer almost entirely to the individual orientation of the panels.

\section{Actual Stage \& Original Contributions}

The orientation principle of the photovoltaic panels is based on the input data referring to the position of the Sun on the sky dome. For the highest conversion efficiency, the sunrays have to fall normal on the receiver (i.e. the photovoltaic panel) so the system must periodically modify its position in order to maintain this relation between the sunrays and the panel. The positions of the Sun on its path along the year represent an input data in designing the tracking system, so the geometrical relationship between the Earth and the Sun has to be considered. The Earth describes along the year a rotational motion following an elliptical path around the sun. During one day, the Earth also spins around its own axis describing a complete rotation, which generates the sunrises and the sunsets. The variation of the altitude of the sun on the celestial sphere during one year is determined by the precession motion, responsible for a declination of the Earth axis in consideration with the plane of the elliptic yearly path.

In these conditions, there are two fundamental ways to track the Sun, by one axis or by two axes; this fact determines two types of tracking mechanisms: single axis, and dual-axis solar trackers. The single-axis tracking mechanisms pivot on their axis to track the sun, facing east in the morning and west in the afternoon. The tilt angle of this axis equals the latitude angle of the loco because this axis has to be always parallel with the polar axis. In consequence for this type of single axis tracker is necessary a seasonal tilt angle adjustment. The dual-axis tracking systems follow combine two rotational motions, so that they are able to follow very precisely the sun path along the period of one year; that's why dual axis tracking systems are more efficient than the single one.

Depending on the mode in which the rotational motions are performed, there are two types of dual-axis tracking systems: polar and azimuthally systems. For the polar systems, there are two independent motions (daily motion and seasonal motion), and this because the main (daily) motion is made by rotating the panel around the polar axis. The azimuthally systems realize the daily motion by rotating the panel around the vertical axis; in this case, it is necessary to continuously combine the vertical rotation with an elevation motion around the horizontal axis, the correlation between motions increasing the complexity of the control process.

The bibliography research reveals a series of aspects and hardships in the literature concerning the tracking systems for PV groups/arrays. In the literature there are no unitary models for the tracking mechanisms of the PV arrays referring to the structural, kinematical and dynamical issues. In the same time, there is no general approach for conceptual design and structural synthesis of these mechanisms. Thereby becomes obviously the necessity a method for the unitary modelling of the tracking mechanisms and according to the strategy proposed by the paper this method is based on the MultiBody Systems (MBS) theory, which facilitates the selfformulating algorithms $[1,2]$.

The issue concerning the control is approached mostly for the tracking systems of the individual panels, using different techniques (closed loop systems with photo sensors, opened loop systems based on astronomical computerized systems, or hybrid combinations), and types of controllers - PID, FNC, FNLC [3-7]. The research is focused mostly on the quantity of the energy achieved by tracking, but there is no evaluation on the energy consumption for orientation. A possible cause of this case is due to the fact that the issue is not approached as an integrated assembly (the mechanical device + the actuating system \& control).

The disposing of the panels in the array topology is approached in the literature exclusively from the point of view of the self-shading avoiding [8]. Complementary our research approaches the issue regarding also the simplification of the solution for transmitting the motion from the driving source to the panels of the array, in order to obtain efficient and feasible solutions. Following this trend, we also investigate a modular approach of the PV array by designing the modules of panels with tracking systems and with the possibility to transmit (or receive) the motion to (from) another module, function of the number of the panels of the array.

Therefore, the paper is approaching the improvement of the efficiency of the photovoltaic conversion by designing \& optimizing a tracking mechanism that simultaneously changes the position of a group of panels. The main task in optimizing the mechanism is to maximize the energetic gain by increasing the solar input and minimizing the energy consumption for tracking. The paper proposes the integration of the mechanic and control components at the virtual prototype level (modelling in the mechatronic concept), which allows performing the energy balance: energy gain by orientation versus energy consumption for realizing the motion. In this way, the physical testing process is greatly simplified, and the risk of the control law being poorly matched to the real system is eliminated.

\section{Case Study}

The application is made for a dual-axis system, at which the driving source for the daily motion is a rotary motor, while the seasonal motion is performed with a linear actuator (fig. 1,a). The motion is transmitted from the motor sources, which directly drive the middle panel of the group, with a multi-parallelogram mechanism (fig. $1, b)$, the revolute axes of the panels being parallel with 
the polar axis. The mechanism is designed for a group of panels disposed in line, but it can be also adapted for other configurations (in the general case, the matrix configuration).

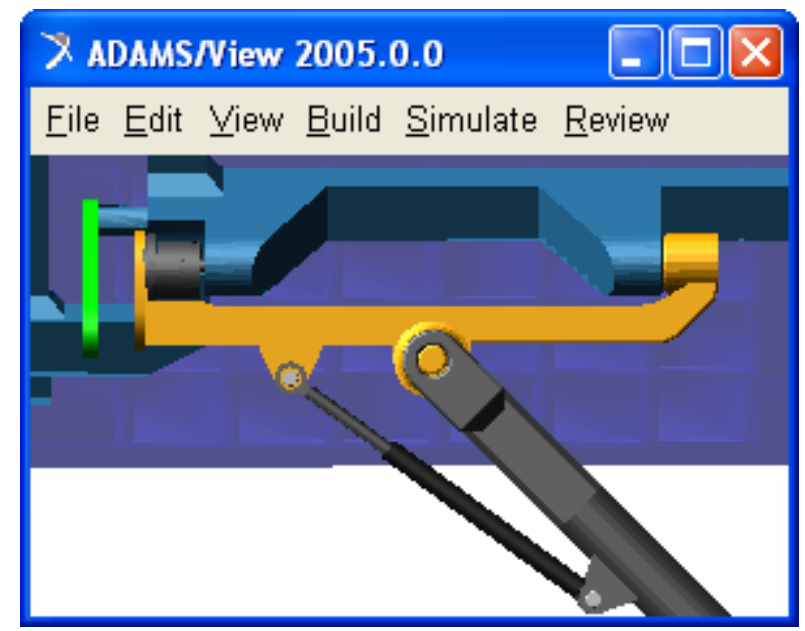

a.

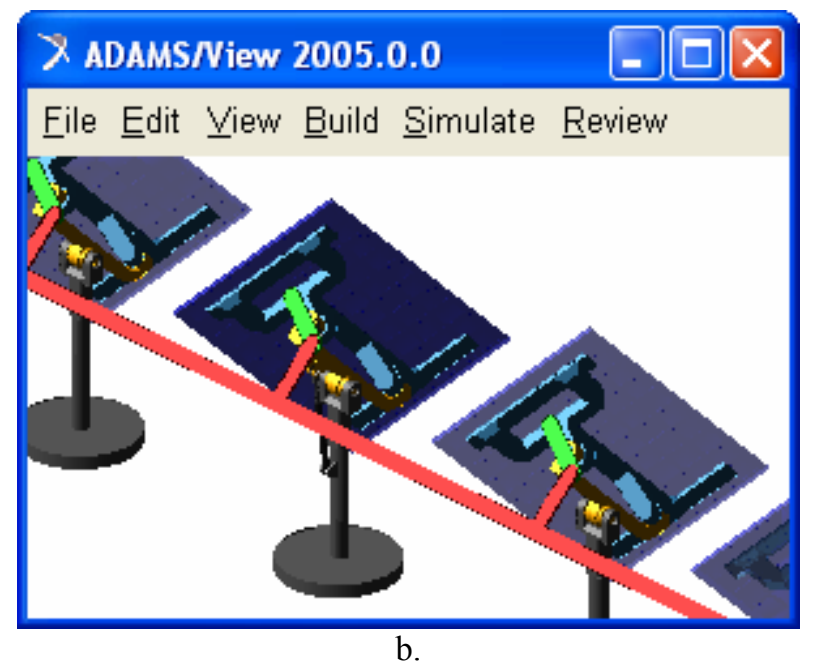

Fig. 1. The virtual prototype of the tracking mechanism

For simulating the behaviour and evaluating the efficiency of the tracking mechanism, we have developed the virtual prototype by using a digital prototyping platform that includes the following software solutions: CAD (CATIA) - to create the solid model of the system, which contains information about the mass \& inertia properties of the parts (bodies), MBS (ADAMS/View) for analyzing, optimizing, and simulating the photovoltaic tracking system, and C\&C (EASY5 \& ADAMS/Controls) - for modelling the control system of the mechatronic product.

The dynamic model of the tracking mechanism takes into consideration the mass forces, the reaction in joints, and the joint frictions, which are modelled by the coefficient of dynamic friction, the friction arm, the bending reaction arm, the radius of the pin, the stiction transition velocity, the maximum stiction deformation, and the preload friction torque in the joint. For blocking the system in the stationary positions between actuatings, when the motors are stopped, the model contains two irreversible transmissions, as follows: worm gearbox - for the rotary motor, and screw-nut mechanism - for the linear actuator. In this way, there is no energy consumption in the stationary positions.

For connecting the MBS mechanical model and the electronic control system, the input \& output parameters have been defined. The motor forces generated by the driving sources represent the input parameters in the mechanical model. The outputs transmitted to the controller are the daily and seasonal angles of the panels. From the controller point of view, for obtaining reduced transitory period and small errors, we used generic control loop feedback mechanisms (PID controllers). For example, in the figure 2 , there is presented the control system of the rotary motor (for the daily motion). In this diagram, the block "MSC.ADAMS" represents the mechanical device model (shown in figure 1).

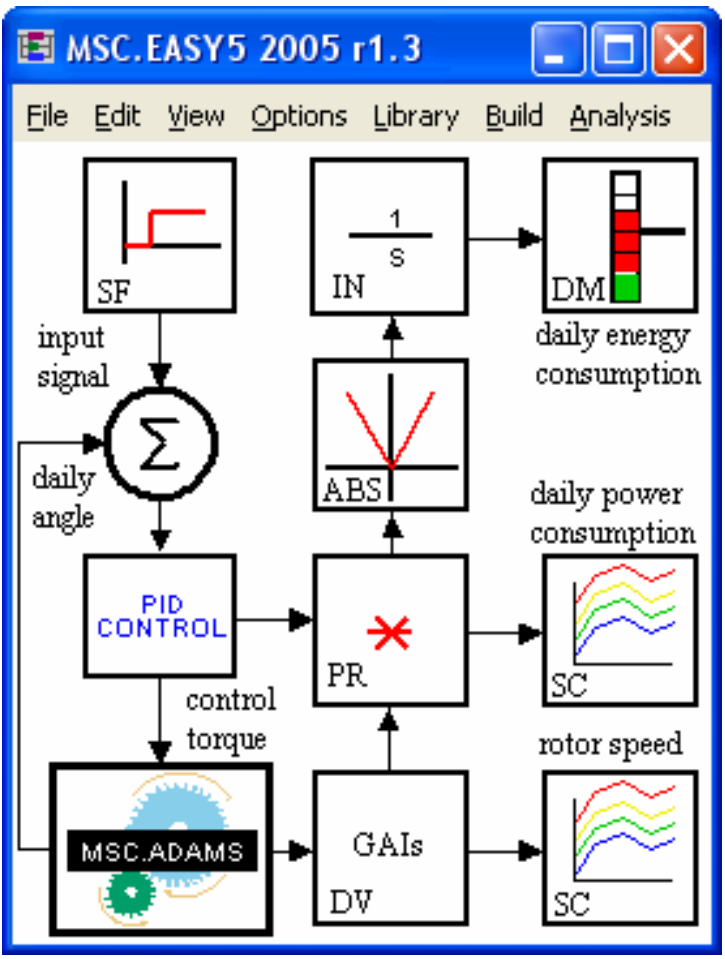

Fig. 2. The control system for the daily motion

The energy produced by the PV group depends on the quantity of incident solar radiation, the panel efficiency, and the number of panels. The incident radiation depends on the direct terrestrial radiation, and the angle of incidence. The direct radiation is empirical established depending on the extraterrestrial radiation, the medium solar constant, the day number during a year, the distortion factor, the solar altitude angle, the solar declination, the latitude angle, the solar hour angle, and the local solar time [9]. The incidence angle is determined from the scalar product of the Sun's ray vector and the normal vector on panel. In this way, we are able to estimate the incident radiation in every day during a year, for different locations, and tracking strategies. The paper presents the exemplification for the summer solstice day, which is relevant for evaluating the energetic efficiency of the photovoltaic system, considering the Braşov geographic area. 
The PV panels can be rotated without brakes during the day-light, or can be discontinuously driven (step-by-step motion), usually by rotating the modules with equal steps at every hour. Obviously, the maximum incident solar radiation is obtained for the continuous motion in the entire angular field (from $-90^{\circ}$ at sunrise, to $+90^{\circ}$ at sunset), but in this case the operating time of the motors is high. In paper, the optimization strategy aims to reduce the angular field of the daily motion and the operating time of the motor source, without significantly affecting the incoming solar energy, and with minimum energy consumption. This strategy involves the identification of the optimum angular field, as well as the optimum actuating time in the step-by-step motion.

For identifying the optimal motion field, we have considered the correlation between the motion amplitude and the local time, for obtaining symmetric revolute motions relative to the solar noon position. The analysis has been performed for the following tracking cases: (a) $\beta^{*} \in\left[-90^{\circ},+90^{\circ}\right] ; \quad$ (b) $\beta^{*} \in\left[-75^{\circ},+75^{\circ}\right] ;$ (c) $\beta^{*} \in\left[-60^{\circ}\right.$, $\left.+60^{\circ}\right]$; (d) $\beta^{*} \in\left[-45^{\circ},+45^{\circ}\right]$; (e) $\beta^{*} \in\left[-30^{\circ},+30^{\circ}\right]$; (f) $\beta^{*} \in[-$ $\left.15^{\circ},+15^{\circ}\right]$; (g) $\beta^{*}=0$ - the fixed photovoltaic panels $\left(\beta^{*}\right.$ is the daily angle of the panels). In this study, we considered that the panels are rotated without brakes.

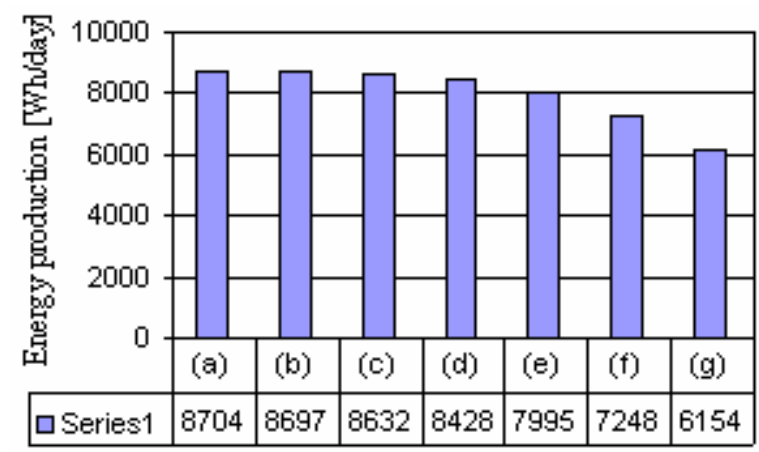

Fig. 3. The energy produced by the PV system.

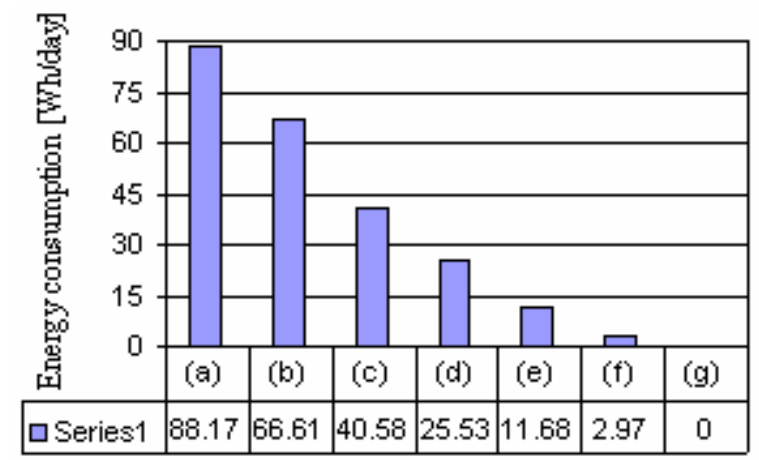

Fig. 4. The energy consumption for tracking.

In this way, we have obtained the incident radiation curves for the considered tracking cases. Taking into account the number of modules in the PV group (the study is made for a string with 5 panels), the active surface of the module $\left(1.26 \mathrm{~m}^{2}\right.$ for each module) and the module's efficiency $(15 \%)$, we have obtained the energy produced by the PV system (fig. 3). Afterwards, the energy consumption for realizing the motion law was determined (fig. 4). In this way, the energy balance was performed, the results being systematized in the figure 5 (the efficiency is relative to the fixed group case). Analyzing these results, we consider that the optimal motion field is $\beta^{*} \in\left[-60^{\circ},+60^{\circ}\right]$, in which there is the best ratio between the energy gain and the operating time of the motor.

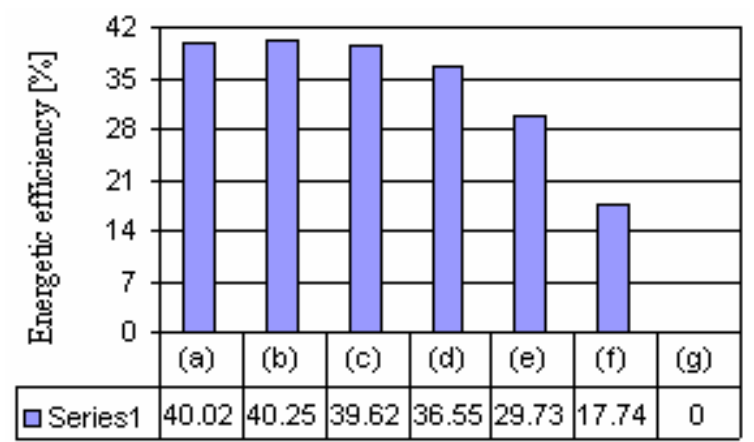

Fig. 5. The energetic efficiency of the tracking system

Afterwards, in the optimal angular field, we have evaluated different step-by-step tracking strategies. The objective is to minimize the operating time, which is important for the durability \& reliability of the tracking system. In these terms, we have developed - analyzed six tracking cases, depending on the number of steps (in consequence, the step dimension $-\Delta \beta^{*}$ ) for realizing the optimum angular field: 12 steps $\left(\Delta \beta^{*}=10^{\circ}\right), 10$ steps $\left(\Delta \beta^{*}=12^{\circ}\right), 8$ steps $\left(\Delta \beta^{*}=15^{\circ}\right), 6$ steps $\left(\Delta \beta^{*}=20^{\circ}\right), 4$ steps $\left(\Delta \beta^{*}=30^{\circ}\right), 2$ steps $\left(\Delta \beta^{*}=60^{\circ}\right)$. In each case, the angular velocity for the motion steps is 10 degrees per minute. For these step-by-step tracking cases, the results are shown in the diagrams from figures 6-8.

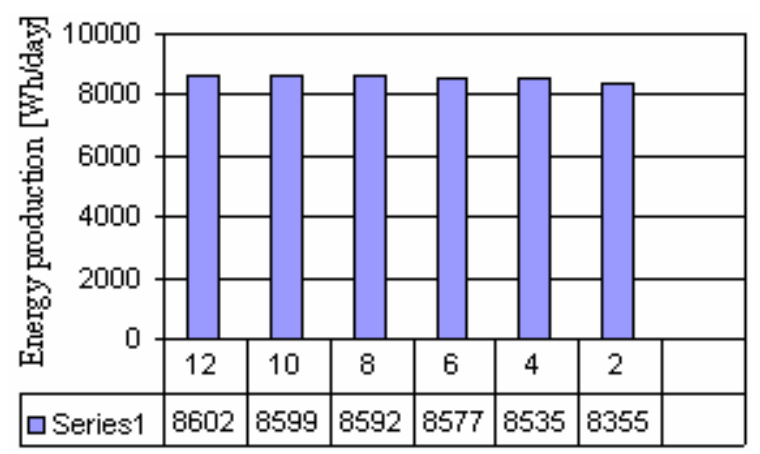

Fig. 6. The energy production for step-by-step motion

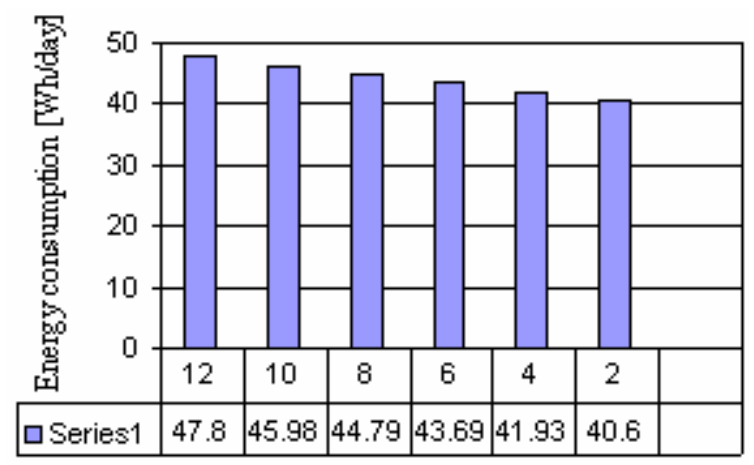

Fig. 7. The energy consumption for step-by-step motion 


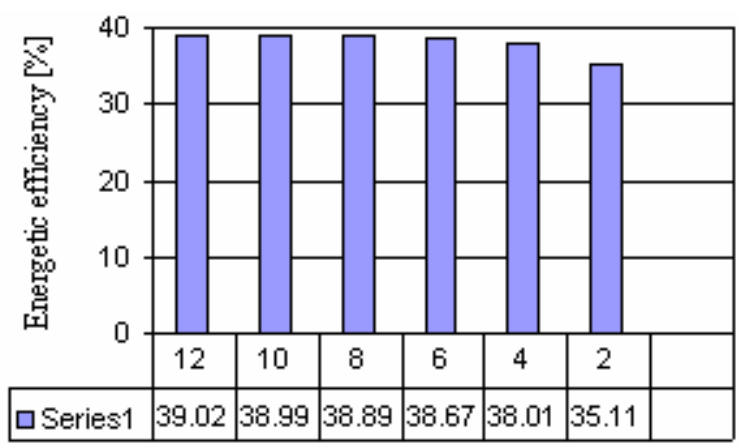

Fig. 8. The efficiency for the step-by-step motion

In this way, we have obtained values closed-by the continuous motion case (see figure 3 - case c), and this demonstrates the viability of the adopted strategy. According with the diagrams from figure 3 (case c) and 7 , the energy consumptions for realizing the step-by-step motion laws are a little bit greater than the energy consumption for the continuous motion, and this because of the over-shootings that appear when the motor is turned-on/off.

\section{Final Remarks}

The application is a relevant example regarding the implementation of the virtual prototyping tools in the design process of the tracking systems. One of the most important advantages of this kind of analysis - simulation is the possibility to perform virtual measurements in any point or area of the tracking system, and for any parameter (motion, force, energy). At the same time, integrating the electronic control system and the mechanical device of the tracking mechanism at the virtual prototype level, the physical testing process is greatly simplified, and the risk of the control law being poorly matched to the real system is eliminated.

The optimization strategy based-on the minimization of the angular field for the daily motion and the minimization of the number of actuating operations leads to an efficient PV system, without developing expensive hardware prototypes. In this way, the behavioral performance predictions are obtained much earlier in the design cycle of the tracking systems, thereby allowing more effective and cost efficient design changes and reducing overall risk substantially.
The tracking system will be manufactured and tested in the Centre Product Design for Sustainable Development from Transilvania University of Braşov, creating a real perspective for the research in the field. This allows a relevant comparison between the virtual prototype analysis and the data achieved by measurements.

\section{Acknowledgements}

This research is financial supported by the Romanian Ministry of Education, in the frame of the CNCSIS research grant, code 892/2007-2008.

\section{References}

[1] E.J. Haug, Computer Aided Kinematics \& Dynamics of Mechanical Systems, Allyn \& Bacon, 1995.

[2] W.O. Schiehlen, Multibody Systems - Roots and Perspectives, Multibody Systems Dynamics, vol. 1 (2), p. 149-188, 1997

[3] S. Abdallah, S. Nijmeh, Two-Axis Sun Tracking with PLC Control, Energy Conversion and Management, n. 45, p. 31-39, 2004.

[4] P. Roth, A. Georgiev, H. Boudinov, Cheap Two Axis Sun Following Device, Energy Conversion and Management, vol. 46 (7-8), p. 1179-1192, 2005.

[5] F.R. Rubio, M. Ortega, F. Gordillo, Application of New Control Strategy for Sun Tracking, Energy Conversion and Management, vol. 48 (7), p. 21742184, 2007.

[6] A. Al-Mohamad, Efficiency Improvements of Photovoltaic Panels using a Sun-Tracking System, Applied Energy, vol. 79 (3), p. 345-354, 2004.

[7] J.A. Chojnacki, Application of Fuzzy Logic Neural Network Controllers in PV Systems, Proc. of the 20th European Photovoltaic Solar Energy Conference, Barcelona, p. 2269-2272, 2005.

[8] P.J. Perez, G. Almonacid, P.G. Vidal, Estimation of Shading Losses in Multi-Trackers PV Systems, Proc. of the 22-nd European Photovoltaic Solar Energy Conference, Milano, p. 2295-2298, 2007.

[9] M. Meliß, Regenerative Energiequellen - Praktikum, Springer-Verlag, 1997.

[10] *** Getting Started using ADAMS v.2005, MSC Software Publisher, Santa Ana, 2005.

[11] *** Getting Started using EASY5 v.2005, MSC Software Publisher, Santa Ana, 2005. 